\title{
Hot Gas and AGN Feedback in Galaxies and Nearby Groups
}

\author{
Christine Jones ${ }^{1}$, William Forman ${ }^{1}$, Akos Bogdan ${ }^{1}$, Scott Randall ${ }^{1}$, \\ Ralph Kraft ${ }^{1}$, and Eugene Churazov ${ }^{2}$ \\ ${ }^{1}$ Center for Astrophysics, \\ 60 Garden Street, Cambridge, MA 02138, USA \\ email: cjones@cfa.harvard.edu, wforman@cfa.harvard.edu, abodgan@cfa.harvard.edu, \\ srandall@cfa.harvard.edu, rkraft@cfa.harvard.edu \\ ${ }^{2}$ Max Planck Institute for Astrophysik, \\ Karl-Schwarzchild-Str. 1., Garching, Germany \\ email: echurazov@mpa-garching.mpg.de
}

\begin{abstract}
Massive galaxies harbor a supermassive black hole at their centers. At high redshifts, these galaxies experienced a very active quasar phase, when, as their black holes grew by accretion, they produced enormous amounts of energy. At the present epoch, these black holes still undergo occasional outbursts, although the mode of their energy release is primarily mechanical rather than radiative. The energy from these outbursts can reheat the cooling gas in the galaxy cores and maintain the red and dead nature of the early-type galaxies. These outbursts also can have dramatic effects on the galaxy-scale hot coronae found in the more massive galaxies. We describe research in three areas related to the hot gas around galaxies and their supermassive black holes. First we present examples of galaxies with AGN outbursts that have been studied in detail. Second, we show that X-ray emitting low-luminosity AGN are present in $80 \%$ of the galaxies studied. Third, we discuss the first examples of extensive hot gas and dark matter halos in optically faint galaxies.
\end{abstract}

Keywords. galaxies: nuclei, galaxies: bulges, galaxies: ISM X-rays: galaxies: clusters

\section{Introduction}

In both galaxies and clusters, X-ray observations of hot gas have been used to determine the overall morphology and characterize the dynamical histories of these systems, as well as to measure the total system mass and, of particular importance to this discussion, to determine the level of recent AGN activity from the galaxy nucleus. While the hot $\mathrm{X}$-ray emitting gas in clusters is the dominant component of the luminous baryonic mass, in galaxies, the hot gas is only a few percent of the stellar mass. While the hot intracluster medium dominates the X-ray emission in clusters, in galaxies, emission from X-ray binaries, coronally active stars and cataclysmic variables, and the AGN can also be important (e.g. Revnivtsev et al. 2006).

\section{SMBH Outbursts in Early-type Galaxies}

In both galaxies and clusters, the hot X-ray emitting gas provides a fossil record of AGN activity and is often the primary evidence of recent AGN activity. Proof of recent AGN outbursts is commonly found through Chandra and XMM-Newton images, which show cavities in $\sim 30 \%$ of galaxies with hot coronae, while $50 \%$ of clusters exhibit such cavities (Dunn and Fabian 2008). From these X-ray observations, the total mechanical power of the AGN outburst can be measured directly from the energy required to inflate 

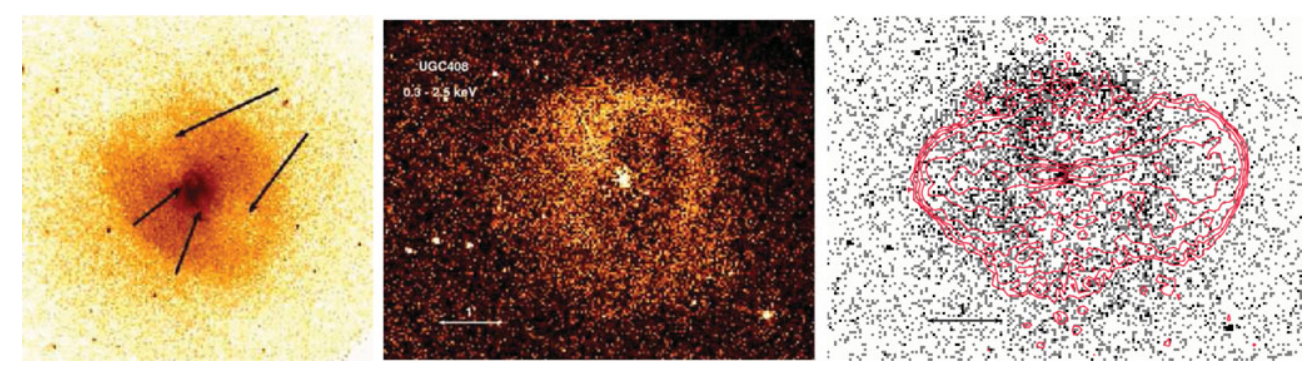

Figure 1. (left) The Chandra image of NGC5813 shows two pairs of cavities (marked with black arrows) indicating multiple episodes of AGN activity. A third pair of cavities lies at slightly larger radii. (center) The Chandra image of the galaxy UGC408 shows a nucleus, jet and bright X-ray rim at $20 \mathrm{kpc}$ radius. (right) UGC408 with $1.4 \mathrm{GHz}$ contours superposed on the X-ray image shows a collimated beam with radio emission extending $\mathrm{E}-\mathrm{W}$, while low frequency radio contours (610 Mhz) show the large extent of the relativistic plasma.

the cavities, while the duration and age of the outburst can be measured from the position and rise time of the bubbles or, in a few systems, from the observations of shocks produced by the outburst (see McNamara \& Nulsen (2007) and references therein). Computations of the radiative cooling and the energy supplied by the AGN through cavities and shocks show that the AGN feedback in early type galaxies is sufficient to balance the radiative losses of the cooling X-ray gas and thus suppresses star formation. The presence of multiple cavities in the hot gas also allows the recurrence frequency of AGN outbursts to be determined.

Many of the central galaxies in groups exhibit the scars of multiple outbursts. Fig. 1 (left) shows three pairs of X-ray cavities in the hot gas around NGC5813; the two inner pairs of cavities are marked with arrows. In addition, NGC5813 has two confirmed sets of shocks, at the locations of sharp brightness discontinuities. The outer shock has a velocity of $\sim 480 \mathrm{~km} \mathrm{~s}^{-1}$ (Mach 1.15). The outburst that produced the middle set of cavities, which are $\sim 5 \mathrm{kpc}$ in diameter and associated with this shock, had a total energy of $10^{55}$ ergs and occurred about 2 Myr ago. Additional information on the NGC5813 outbursts can be found in Randall et al. (2011).

For the galaxy UGC408 (NGC 193), the combination of radio and X-ray observations suggest two episodes of AGN activity (see Fig. 1 center and right). The X-ray images show a $20 \mathrm{kpc}$ radius ring of enhanced X-ray emission surrounding a large X-ray cavity centered on the nucleus. This cavity is filled with radio plasma emitting at $610 \mathrm{MHz}$. However the $1.4 \mathrm{GHz}$ radio emission is significantly more collimated and likely arises from a second outburst. An X-ray jet is associated with the eastern radio emission.

\section{X-ray Emission from the Supermassive Black Holes in the Nuclei in Early-type Galaxies}

While observations of cavities or shocks in the hot gas can measure the energy of an AGN outburst, detection of point-like X-ray emission in the central cores of galaxies identifies the presence and the precise location of the supermassive black hole, as well as measuring the low level of radiative power arising from the black hole at the present epoch. Fig. 2 (left) plots the X-ray emission from galaxy nuclei for a sample of about 200 early-type galaxies observed with Chandra. We detect X-ray emission from the supermassive black hole for approximately $80 \%$ of the galaxies with hot corona. The black hole X-ray luminosities range from $10^{38}$ to $10^{41} \mathrm{ergs} \mathrm{s}^{-1}$. We use each galaxy's velocity dispersion to calculate the mass of the black hole and then compute the ratio of the black 

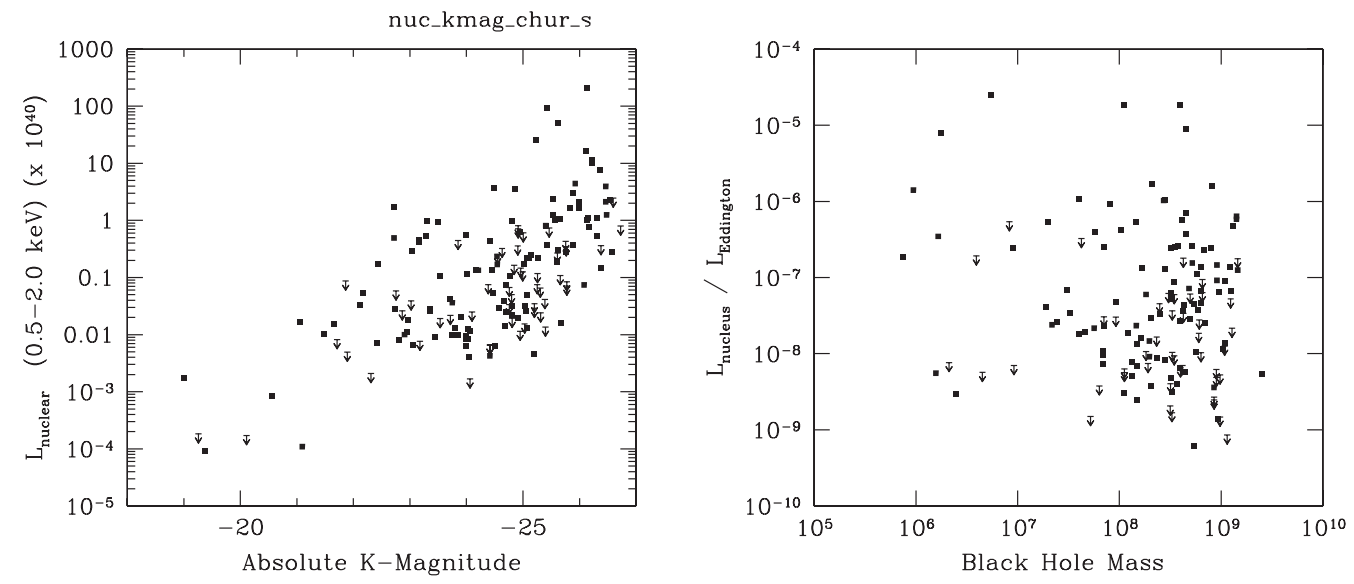

Figure 2. (left) The X-ray luminosities of the nuclear emission in a sample of early-type galaxies plotted against each galaxy's K magnitude. Emission from more than $80 \%$ of the supermassive black holes in galaxies with gas coronae are detected in X-rays with Chandra. (right) The Eddington ratio for supermassive black holes, where the mass of the black hole is estimated from the velocity dispersion and the nuclear luminosity from X-ray measurements.

hole's observed luminosity to its possible Eddington luminosity. These Eddington ratios, shown on the right in Fig. 2, in these low luminosity AGN range from $\sim 10^{-5}$ to $\sim 10^{-9}$. The radiative luminosities of these supermassive black holes are much smaller than their mechanical energies, when measured through observations of X-ray cavities. Thus, the present epoch AGN mode is one of mechanical power, not radiative power (see Churazov et al. 2005 for a discussion of the AGN transition from radiatively bright to radiatively faint and mechanically powerful.)

\section{Dark Matter Haloes, Stellar Bulges and the Growth of Supermassive Black Holes}

Although galaxies with large stellar bulges are observed to harbor supermassive black holes (e.g. Gultekin et al. 2009), recently, examples of galaxies have been identified which do not have large stellar bulges, but still harbor supermassive black holes. In particular, the lenticular galaxy NGC4342 hosts an unusually massive black hole, compared to its low bulge mass (Cretton \& van den Bosch (1999)). As illustrated in Fig. 3, NGC4342 also hosts an X-ray bright halo of hot gas that extends more than $10 \mathrm{kpc}$, significantly broader than its stellar bulge. NGC4342's diffuse X-ray luminosity is also significantly higher than generally observed for galaxies with bulges of similar stellar mass (Bogdan et al. 2012a). For NGC4342, the mass of the hot gas and the dark halo mass, inferred from the X-ray observations, are similar to those found for more optically luminous early-type galaxies.

Similar results also have been found for the elliptical galaxy NGC4291 (Bogdan et al. 2012b). The presence of massive dark matter halos, along with supermassive black holes in their cores $\left(4 \times 10^{8} M_{\odot}\right.$ for NGC4342 and $7 \times 10^{8} M_{\odot}$ for NGC4291; Gultekin et al. 2009), but low stellar masses in their bulges suggest that it is the total dark matter halo that plays the fundamental role in governing black hole growth. Bodgan et al. also conclude, based on their results for NGC4342 and NGC4291, that supermassive black holes and galaxy stellar bulges may not grow in tandem, but instead the earlier formation 

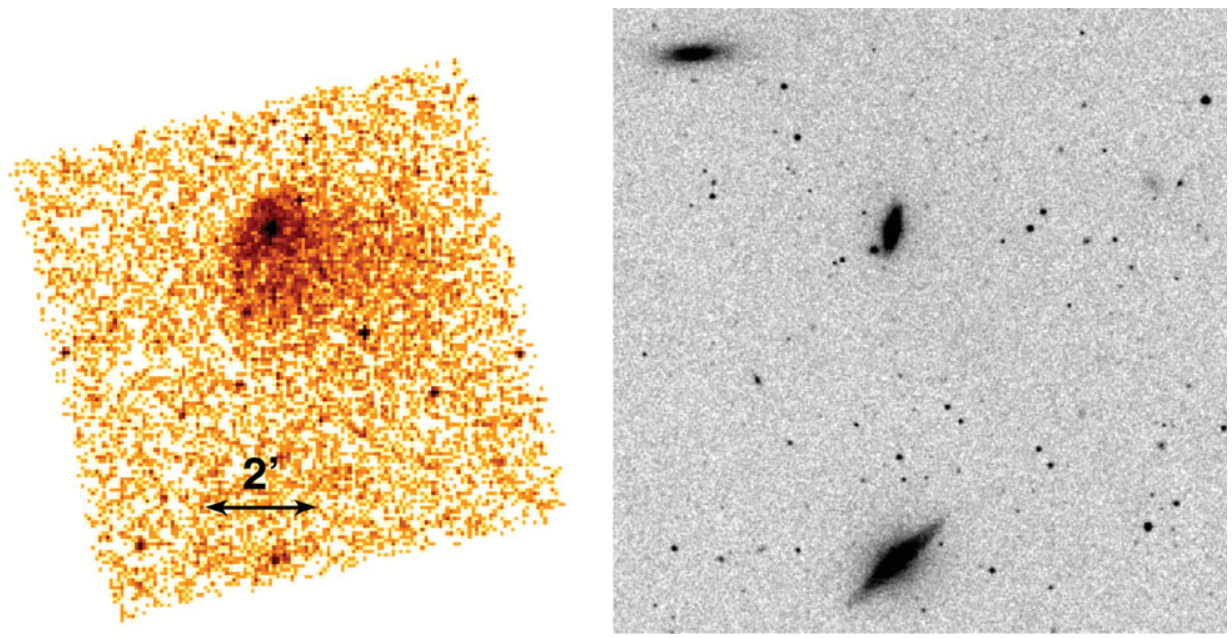

Figure 3. (left) Diffuse X-ray emission from NGC4342 extends beyond $10 \mathrm{kps}$, much broader than the stellar bulge, shown on the same spatial scale, in the right panel. The sharp edge, seen in the diffuse X-ray emission, northeast of the core, results from the motion of NGC4342 through the surrounding group gas. For details see Bogdan et al. (2012a).

and growth of supermassive black holes can suppress star formation, limiting the growth of the stellar bulge.

\section{Implications}

We reviewed three areas related to hot coronae around early-type galaxies and their supermassive black holes. First, we noted that the cavities and shocks produced in the hot gas by AGN outbursts can be used to measure the mechanical energy and age of the outbursts. Second we showed that low luminosity X-ray AGN are common in apparantly normal early-type galaxies in the local Universe, and third we discussed two galaxies with small stellar bulges that harbor supermassive black holes, along with extended hot coronae and dark matter halos.

We are greatful to many of our colleagues for useful discussions related to this work, particularly Larry David, Paul Nulsen, Marie Machacek and Ming Sun. Support was received from the Smithsonian Institution, the Chandra X-ray Center and the Max Planck Institute for Astrophysik.

\section{References}

Bogdan, A., Forman, W., Kraft, R., Jones, C., Blom, C., Randall, S., Zhang, Z., Zhuravleva, I., Churazov, E., Li, Z., Nulsen, P., Vikhlinin, A., \& Schindler, S. 2012, ApJ, 755, 25

Bogdan, Forman, W., Zhuravleva, I., Mihos, C., Kraft, R., Harding, P., Guo, Q., Li, Z., Churazov, E., Vikhlinin, A., Nulsen, P., Schindler, S., \& Jones, C. 2012, ApJ, 753, 140

Churazov, E., Sazonov, S., Sunyaev, R., Forman, W., Jones, C., \& Bohringer, H. 2005, MNRAS, 363, L91

Cretton, N. \& van den Bosch, F. C. 1999, ApJ, 514, 704

Dunn, R. J. H. \& Fabian, A. C. 2008, MNRAS, 385, 757

Gultekin, K., et al. 2009, ApJ, 695, 1577

McNamara, B. R. \& Nulsen, P. E. J. 2007, ARA\&A, 45, 117

Randall, S., et al. 2011, ApJ, 726, 86

Revnivtsev, M., Sazonov, S., Gilfanov, M., Churazov, E., \& Sunyaev, R. 2006, A\&A, 452, 169 